\title{
ATLAS search for dark matter produced in association with a hadronically decaying vector boson
}

\author{
Wei Wang*, on behalf of the ATLAS Collaboration \\ School of Physics, Nanjing University \\ Institute of Physics, Academia Sinica \\ E-mail: wei.wangecern.ch
}

In this report we present the latest results from the ATLAS experiment on the search of dark matter production in association with a hardronically decaying vector boson. The search is performed on proton-proton collisions produced by the Large Hadron Collider with the first two years of Run 2 data taking by ATLAS. The simplified vector-mediator model and the invisible Higgs model are considered in this search with a $W$ or $Z$ boson in the final state, while the dark-fermion model and dark-Higgs model are considered where a potentially new vector boson $Z^{\prime}$ is presented. No significant excess over the Standard Model prediction is observed. The results are interpreted in terms of constraints on the parameter space of the simplified vector-mediator model, limits on the branching ratio of Higgs boson decay into dark matter particles, as well as the highest possible production cross section of dark-fermion and dark-Higgs models.

2nd World Summit: Exploring the Dark Side of the Universe

25-29 June, 2018 - EDSU2018

University of Antilles, Pointe-à-Pitre, Guadeloupe, France

\footnotetext{
* Speaker.
} 


\section{Introduction}

Dark matter (DM), as indicated by many cosmological observations, is believed to be a significant component of our universe. However its exact nature and its connection to the Standard Model (SM) particle physics remain unknown. Many experiments have been designed to search for the DM, including indirect searches by looking for signs of DM annihilation or decays, direct searches where DM interacts with SM particles, as well as collider searches that DM may be produced through SM particle interactions.

One of the leading DM candidates, the weakly interacting massive particles (WIMPs), can be produced in proton-proton ( $p p$ ) collisions at the Large Hadron Collider (LHC) [1], and detected by the general-purpose detector, ATLAS [2], at the LHC. The ATLAS detector has a forwardbackward symmetric cylindrical geometry, consisting an inner tracking detector, electromagnetic and hadronic calorimeters and a muon spectrometer surrounding the interaction point. A typical DM signature in the ATLAS detector is a large missing transverse momentum, $E_{T}^{\text {miss }}$, from a pair of DM particles which are recoiling against one or more SM particles.

The latest results from the ATLAS experiment on the search of DM production in association with a hardronically decaying vector boson is presented in this report [3], including the search for the DM that is produced together with a $W$ or $Z$ boson (mono- $V$ analysis), a SM-like Higgs boson decaying into a pair of DM particles ( $H \rightarrow$ invisible analysis), and the search of DM production in association with a potentially new vector boson $Z^{\prime}$ (mono- $Z^{\prime}$ analysis). The analysis uses LHC $p p$ collision data at a centre-of-mass energy of $13 \mathrm{TeV}$ collected by the ATLAS experiment in 2015 and 2016, corresponding to a total integrated luminosity of $36.1 \mathrm{fb}^{-1}$.

\section{Signal models}

Four signal models are considered in this analysis. The simplified vector-mediator model discribes the DM produced from the exchange of a vector mediator $Z^{\prime}$, shown in figure 1a. The invisible Higgs model introduces a SM-like Higgs boson decaying into a pair of DM particles. All the SM Higgs production modes are considered. The one with final state closest to the mono$V$ signature is the production in associated with a vector boson, shown in figure $1 \mathrm{~b}$. There're two signal models for the mono- $Z^{\prime}$ analysis, One is the dark-fermion model, where the $Z^{\prime}$ boson couples to a heavier dark-sector fermion $\chi_{2}$ and a lighter DM candidate $\chi_{1}$, shown in figure $1 \mathrm{c}$. The other is the dark-Higgs model, where a dark-sector Higgs boson is radiated from $Z^{\prime}$ and decays into DM particles, shown in figure $1 \mathrm{~d}$.

To simulate the signal events, several choices of the mass and coupling strength samples are made. For the mono- $Z^{\prime}$ analysis, samples are further separated into light and heavy dark-sector scenarios depending on the mass of DM or the mass of dark-Higgs for each $Z^{\prime}$ mass point.

\section{Event selection}

In this analysis, the DM particles will pass through the ATLAS detector, leaving a signature of $E_{T}^{\text {miss }}$, while the vector boson decay hardronically and are detected as jets in the calorimeter. Depending on how boosted the vector boson is, signal regions are defined in the merged topology 


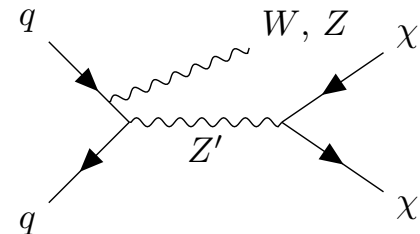

(a) simplified vector-mediator model

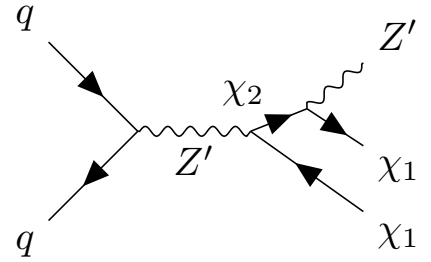

(c) dark-fermion model

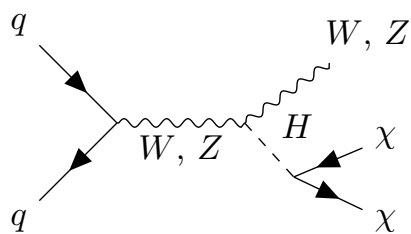

(b) invisible Higgs model

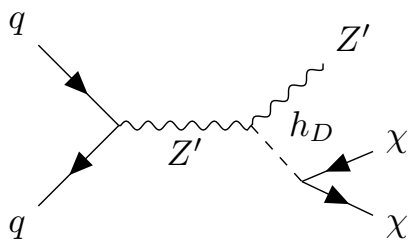

(d) dark-Higgs model

Figure 1: Feynman diagrams of four signal models [3]

with $E_{T}^{\text {miss }}$ above $250 \mathrm{GeV}$ and one large radius jet, or, if not passed the merged selection, the resolved topology with $E_{T}^{\text {miss }}$ above $150 \mathrm{GeV}$ and two small radius jets. Inside each topology, events are further categorised into 0,1 and 2 b-jet regions to gain sensitivity for $Z$ to $b \bar{b}$ decay. To enhance the sensitivity in regions around the mass peak, we also require the events to be inside a dedicated mass window of the vector boson for each signal sample.

The dominant backgrounds inside the signal regions are $Z+$ jets, $W+$ jets, and $t \bar{t}$, contributing $93 \%$ of the total background. Several control regions with leptons are defined in order to constrain the backgrounds, targeting $W+$ jets in one lepton region, $Z+j e t s$ in two lepton region and $t \bar{t}$ in 1 or $2 \mathrm{~b}$-jets categories of both one or two lepton region. The selection of control regions is kept as similar as possible to the signal regions in order to have a similar phrase space. A dedicated control region is also introduced for multijet background. The sideband of the mass window in zero lepton region works as a validation region for all the backgrounds.

\section{Results}

To interpret the observation of the search, a profile likelihood fit to the data is performed on the discriminant variable $E_{T}^{\text {miss }}$ distribution. The parameter of interest is signal strength $\mu$, while background normalisation and systematics uncertainties are evaluated and enter the fit as nuisance parameters. Figure 2 are two examples of the $E_{T}^{\text {miss }}$ distribution after the fit in resolved topology for mono- $V$ signal and merged topology for mono- $Z^{\prime}$ signal.

No significant excess over the SM prediction is observed. We set limit on the signal strength at $95 \%$ confidence level for each signal model and interpreted them as limits on DM and mediator mass for mono- $V$ analysis, branching ratio of Higgs to DM decay for $H \rightarrow$ invisible analysis, and cross section for mono- $Z^{\prime}$ analysis.

Figure 3 shows the exclusion of mono- $V$ model in the mediator mass versus DM mass phase space, where mediator mass up to $650 \mathrm{GeV}$ are excluded for DM mass up to $250 \mathrm{GeV}$. The upper limit on the branching ratio of Higgs to invisible decay is set to be 0.83 for observed and 0.58 for 


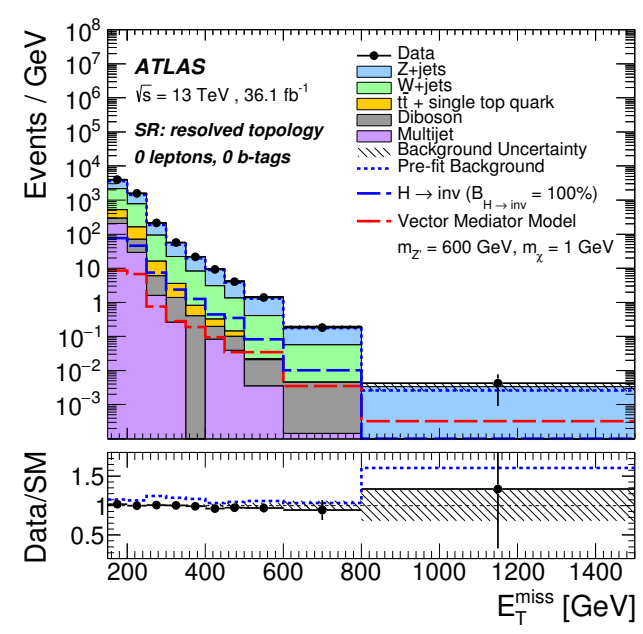

(a)

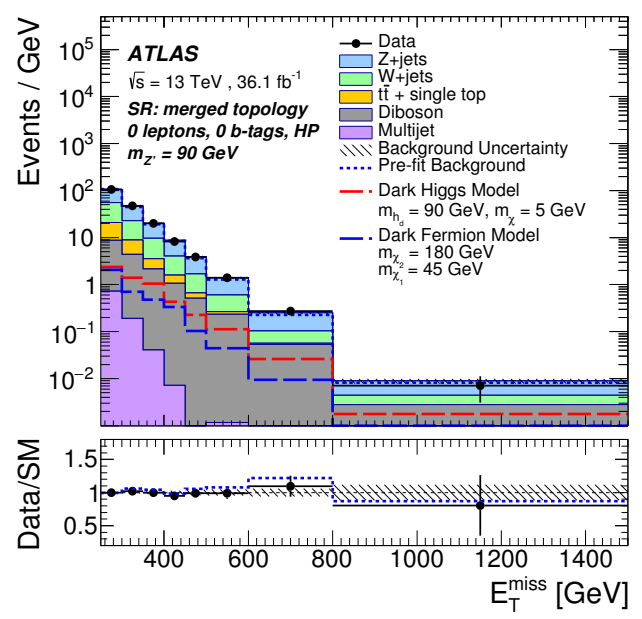

(b)

Figure 2: The observed (dots) and expected (histograms) distributions of missing transverse momentum [3] in the (a) mono- $V$ signal region with the resolved event topology after the profile likelihood fit (with $\mu=0$ ) and (b) mono- $Z^{\prime}$ signal region with $m_{Z^{\prime}}=90 \mathrm{GeV}$ and the merged event topology after the profile likelihood fit (with $\mu=0$ )

expected. Figure 4 shows the cross section limit for mono- $Z^{\prime}$ analysis with respect to mass of $Z^{\prime}$ for dark-fermion and dark-Higgs models in light and heavy dark-sector scenarios.

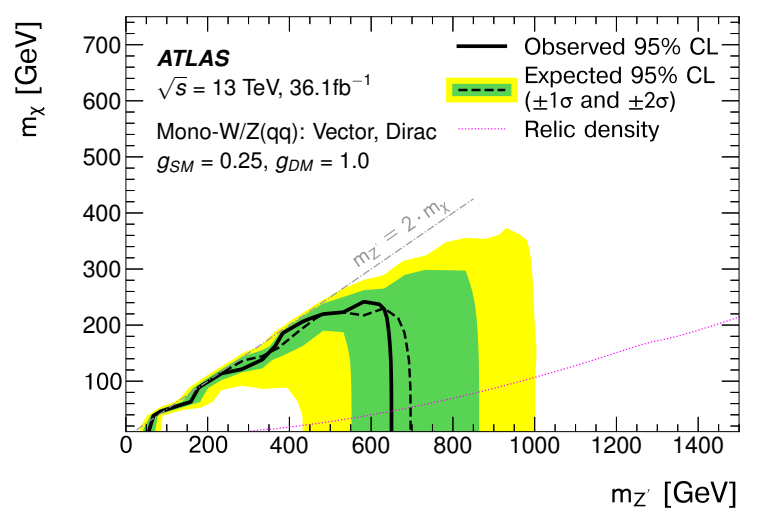

Figure 3: The exclusion contours at 95\% CL for for the combined mono- $V$ search in the simplified vector-mediator model with Dirac DM particles and couplings of gSM $=0.25$ and $\mathrm{gDM}=1$. [3]

\section{Summary}

LHC and ATLAS detector provide a good opportunity of searching for DM produced in $p p$ collisons, with a typical signature of large $E_{T}^{\text {miss }}$ in the detector. A search of DM has been performed in ATLAS experiment in events with a hadronically decaying vector boson and large $E_{T}^{\text {miss }}$. Results are in agreement with SM and are translated into exclusion limits on DM pair production. Mediator 


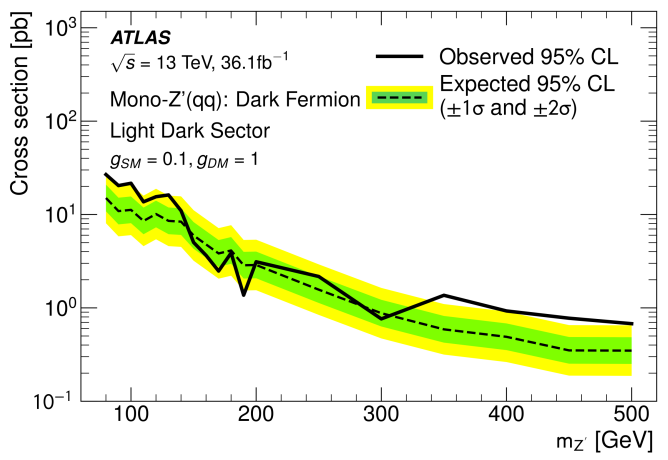

(a)

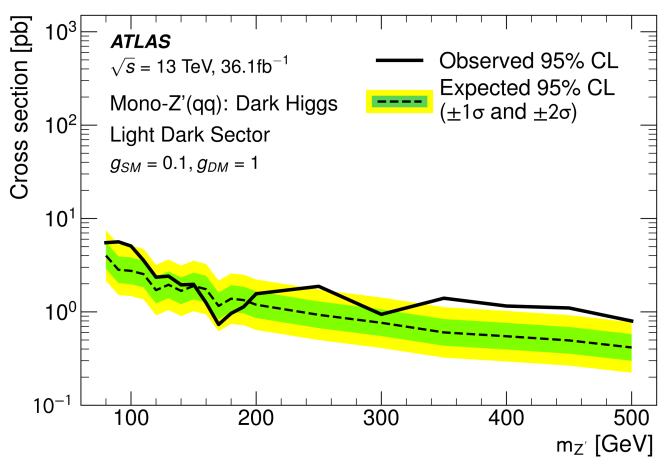

(c)

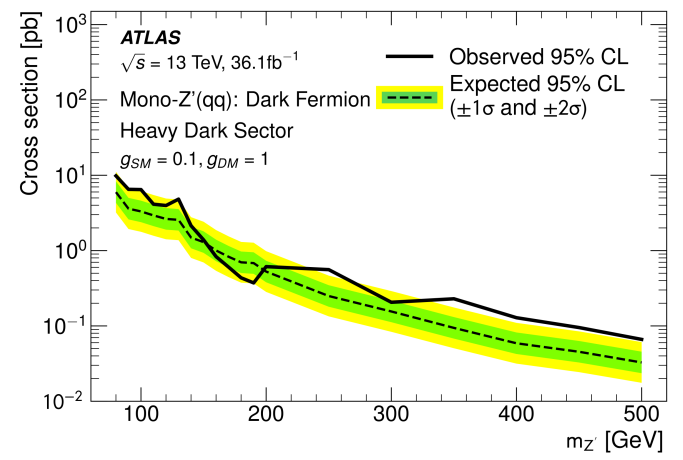

(b)

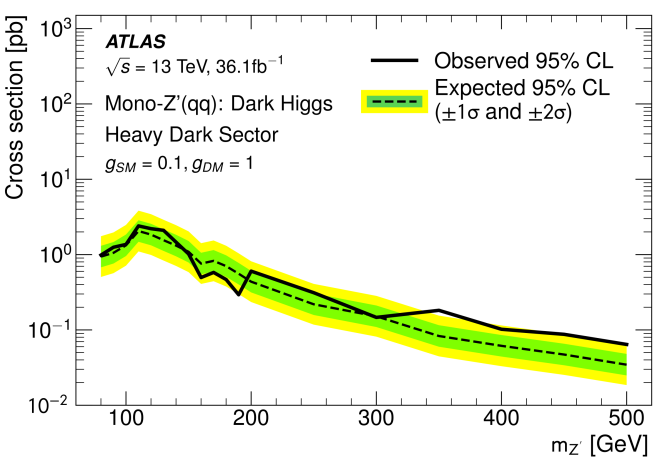

(d)

Figure 4: Upper limits at 95\% CL on the cross section times the branching ratio $\operatorname{Br}\left(Z^{\prime} \rightarrow q^{\prime} q\right)$ in mono- $Z^{\prime}$ models as a function of the mediator mass, $m_{Z^{\prime}}$, for the dark fermion model [3] in the (a) light and (b) heavy dark-sector scenario, as well as the dark Higgs model in the (c) light and (d) heavy dark-sector scenario.

mass up to $650 \mathrm{GeV}$ is excluded for DM mass up to $250 \mathrm{GeV}$ in the simplified vector-mediator model. An upper limit of 0.83 is set on the branching ratio of Higgs boson decaying into a pair of DM particles. Cross section exclusion for dark-fermion and dark-Higgs model are also set in light and heavy dark-sector scenarios with respect to $Z^{\prime}$ mass.

\section{References}

[1] L. Evans and P. Bryant (editors), LHC Machine, JINST 3 (2008) S08001.

[2] ATLAS Collaboration, The ATLAS Experiment at the CERN Large Hadron Collider, JINST 3 (2008) S08003.

[3] ATLAS Collaboration, Search for dark matter in events with a hadronically decaying vector boson and missing transverse momentum in pp collisions at $\sqrt{s}=13 \mathrm{TeV}$ with the ATLAS detector, arXiv:1807.11471[hep-ex]. 